\title{
Stimulus and response conflict processing during perceptual decision making
}

\author{
Carter Wendelken \\ University of California, Berkeley, California \\ JOCHEN DITTERICH \\ University of California, Davis, California \\ Silvia A. Bunge \\ University of California, Berkeley, California \\ AND \\ Cameron S. Carter \\ University of California, Davis, California
}

\begin{abstract}
Encoding and dealing with conflicting information is essential for successful decision making in a complex environment. In the present fMRI study, stimulus conflict and response conflict are contrasted in the context of a perceptual decision-making dot-motion discrimination task. Stimulus conflict was manipulated by varying dot-motion coherence along task-relevant and task-irrelevant dimensions. Response conflict was manipulated by varying whether or not competing stimulus dimensions provided evidence for the same or different responses. The right inferior frontal gyrus was involved specifically in the resolution of stimulus conflict, whereas the dorsal anterior cingulate cortex was shown to be sensitive to response conflict. Additionally, two regions that have been linked to perceptual decision making with dot-motion stimuli in monkey physiology studies were differentially engaged by stimulus conflict and response conflict. The middle temporal area, previously linked to processing of motion, was strongly affected by the presence of stimulus conflict. On the other hand, the superior parietal lobe, previously associated with accumulation of evidence for a response, was affected by the presence of response conflict. These results shed light on the neural mechanisms that support decision making in the presence of conflict, a cognitive operation fundamental to both basic survival and high-level cognition.
\end{abstract}

Cognitive control, or the capacity to modulate or manipulate bottom-up neural processes in the pursuit of high-level goals, is a central feature of high-level cognition. Cognitive control is often essential for decision making in a complex environment and is especially important whenever features of the environment pose challenges to the achievement of a goal. Response conflict and stimulus conflict, both of which demonstrate the need to select between competing representations, are two such challenges. We present here a novel fMRI experiment designed to examine both response conflict and stimulus conflict during perceptual decision making.

Response conflict occurs when simultaneous neural signals support competing response alternatives. It is hypothesized that the presence of response conflict leads to the engagement of top-down cognitive control (Botvinick, Braver, Barch, Carter, \& Cohen, 2001; Kerns et al., 2004). In particular, fMRI studies point to a role for the dorsal anterior cingulate cortex (dACC) in the detection of response conflict (Carter et al., 1998), and to the dorsolateral prefrontal cortex (DLPFC) in its resolution (Egner \& Hirsch, 2005; Kerns et al., 2004; MacDonald, Cohen, Stenger, \& Carter, 2000).

In contrast to response conflict, stimulus conflict occurs when the presence of task-irrelevant perceptual information has a negative impact on the processing of taskrelevant perceptual information (Kornblum, 1994). Thus, stimulus conflict increases with the increasing perceptual strength of task-irrelevant information. Conversely, stimulus conflict should increase with the decreasing perceptual strength of task-relevant information, since in this case reduced perceptual strength is associated with reduced robustness to interference. As is the case with response conflict, stimulus conflict can lead to the engagement of top-down control processes that serve to limit its negative impact on performance (Egner, Delano, \& Hirsch, 2007; Verbruggen, Notebaert, Liefooghe, \& Vandierendonck, 2006). 
Recent imaging studies of stimulus conflict, and other forms of nonresponse conflict, have yielded mixed results. One study involved a letter flanker task, in which multiple letters were mapped to each response option, and stimulus conflict (but not response conflict) was said to be present when the flanker and distractor were different letters that mapped to the same response (van Veen, Cohen, Botvinick, Stenger, \& Carter, 2001). The left inferior frontal gyrus (IFG) was the only region activated here by stimulus conflict. In a second study, involving the Stroop task, the left prefrontal cortex (PFC) was associated with nonresponse conflict (involving color words not linked to any response option), whereas the right PFC and the anterior cingulate cortex (ACC) were associated with response conflict (Milham et al., 2001). A third study, also involving the Stroop task, demonstrated the involvement of the inferior parietal cortex in stimulus conflict (when an incongruent color word mapped to a correct response) and PFC and ACC involvement in both stimulus conflict and response conflict (van Veen \& Carter, 2005). In a fourth study, local and global information (large letters made up of smaller letters) could be in conflict at the stimulus level only or at the response level as well; activation of the middle frontal gyrus and dACC was observed for stimulus conflict, although the latter was activated only for local targets with global distractors (Weissman, Giesbrecht, Song, Mangun, \& Woldorff, 2003). In a fifth study, involving a variant of the Simon task, simultaneous stimulus and response conflict resulted in activation of the inferior parietal lobe (IPL) relative to response conflict alone (Liu, Banich, Jacobson, \& Tanabe, 2004). Finally, in a sixth study, a variant of the Simon task was employed wherein judgments had to be made about the color and motion of two visual stimuli. Stimulus conflict was manipulated by varying the salience of the irrelevant dimension (Liston, Matalon, Hare, Davidson, \& Casey, 2006); in this case, the superior parietal lobe (SPL) was activated for stimulus conflict, along with parts of the PFC (but not the IFG).

It is important to note that in these prior investigations of stimulus conflict, the mapping between stimulus and response depended on a memorized rule. Thus, in a situation with competing stimuli, there is a likelihood that multiple response rules may be accessed, and errors in rule lookup (leading to de facto response conflict) are possible. One goal of the present study was to avoid this problem by utilizing a natural stimulus-response mapping. In addition, none of these prior studies involved separately manipulating salience of both the relevant and irrelevant dimensions. Doing so may be important in order to tease apart effects of stimulus conflict from effects due to perceptual strength; this was another goal of our investigation of stimulus conflict. More broadly, given the current uncertain state of the literature with regard to stimulus conflict, the first major aim of the present study was to clearly compare and contrast the neural correlates of stimulus conflict with the neural correlates of response conflict.

Both stimulus conflict and response conflict can affect the ability to make decisions. In fact, cognitive control mechanisms that allow one to deal with conflict can be essential for effective decision making in many circumstances (Wendelken \& Shastri, 2005). To understand the impact of cognitive control on decision making, it is helpful to first have an understanding of the bottom-up decision processes to which control might be applied. The study of perceptual decision making has focused on this problem. Perceptual decision making involves making simple response decisions (e.g., left or right buttonpress) on the basis of an interpretation of a perceptual stimulus. Perceptual decision tasks have been used to explore the path of information flow in neural circuits, as low-level perceptual information is transformed into high-level percepts, response options, and ultimately into motor behavior.

For one well-studied perceptual decision task, dotmotion discrimination, it has been shown in macaque monkeys that two brain regions play a particularly important role: The middle temporal (MT) area encodes motion direction and strength, and the lateral intraparietal (LIP) area represents accumulated evidence during the decision-making process (Ditterich, Mazurek, \& Shadlen, 2003; Roitman \& Shadlen, 2002; Salzman, Murasugi, Britten, \& Newsome, 1992; Shadlen \& Newsome, 2001). In the dot-motion discrimination task used for these studies, the subject (monkey) observes a field of moving dots in which there is coherent motion in one direction (and random motion in every other direction). Upon determining the direction of coherent motion, the subject indicates this direction (e.g., via saccade). The difficulty of the task is largely determined by the strength of motion coherence, which can vary from trial to trial. The idea that the MT area encodes motion direction (Dubner \& Zeki, 1971; Zeki, 1974) is supported by the finding that microstimulation of this area can influence the interpretation of dot-motion stimuli (Ditterich et al., 2003; Salzman et al., 1992). In related studies, the firing of LIP neurons reflected the accumulation of evidence supporting a particular response, indicating that this region participates in the decision process (Roitman \& Shadlen, 2002; Shadlen \& Newsome, 2001).

Despite the detailed characterization of monkey MT and LIP areas in the context of perceptual decision making, little is known about how these regions and their human homologues operate when the decision task requires cognitive control. Thus, a second aim of the present study was to characterize the operation of these regions in the presence of stimulus conflict and response conflict.

\section{Task Design}

We sought to explore the interaction between perceptual decision making and cognitive control in the context of both stimulus conflict and response conflict. In order to do this, we separately manipulated stimulus conflict and response conflict while human subjects performed a dotmotion discrimination perceptual decision-making task. The basic dot-motion discrimination task was modified in several ways: First, coherent motion was always presented in two directions (one vertical and one horizontal) rather than in one direction; second, the two response targets on each trial were placed in opposite corners of the screen, 
so that each target could be associated with two potential directions of motion; third, subjects were instructed to attend to a particular dimension of motion (vertical or horizontal) on each trial. In addition, two levels of motion coherence (low, 10\%; high, 20\%) were utilized in both dimensions.

Stimulus conflict was manipulated by varying the coherence of relevant versus irrelevant dimensions. We chose to separately manipulate relevant and irrelevant motion coherence in order to distinguish between effects of stimulus conflict and effects of percept strength. A given trial could have high motion coherence in both dimensions (HH), low motion coherence in both dimensions (LL), high coherence in the relevant dimension but low coherence in the irrelevant dimension (HL), or low coherence in the relevant dimension but high coherence in the irrelevant dimension (LH). According to our working definition of stimulus conflict, both decreases in coherence along the relevant dimension and increases in coherence along the irrelevant dimension increase the level of stimulus conflict. The level of stimulus conflict is highest when motion coherence along the irrelevant dimension is stronger than motion coherence along the relevant dimension (LH; a.k.a. high stimulus conflict), and lowest when the reverse situation holds (HL; a.k.a. low stimulus conflict). Those conditions with equivalent motion coherence on both dimensions (LL, HH; a.k.a. medium stimulus conflict) should represent intermediate, although not necessarily equivalent, levels of stimulus conflict. Because we can neither equate the two medium conditions nor definitively order them with respect to each other, we do not consider this to be a parametric design. One might define stimulus conflict differently here-for example, as the product of relevant and irrelevant percept strengths - but the present definition has the advantage that increasing stimulus conflict should be associated with increasing demand for cognitive control.

Response conflict was manipulated by varying whether the two directions of dot motion on a given trial were associated with the same response target (congruent, response conflict absent) or with two different response targets (incongruent, response conflict present). Thus, in our $2 \times 2 \times 2$ factorial design, each trial was associated with three primary factors: response conflict, relevant dimension motion coherence, and irrelevant dimension motion coherence. The eight experimental conditions that result are listed in Table 1.

\section{Modeling and Predictions}

A mathematical model of bottom-up processing during perceptual decision making in the presence of conflict was developed, and this model helped to guide our interpretation of the experimental results. This model is an expansion of the decision model developed in the course of investigating dot-motion discrimination between two alternatives without interfering sensory information, adding to the earlier model a capacity to deal with competing perceptual inputs. For the basic two-alternative forced choice (2AFC) random-dot motion direction discrimination task, it has been demonstrated that the probability of making a correct choice is well described by the logistic function (Palmer, Huk, \& Shadlen, 2005)

$$
P(\text { correct })=\frac{1}{1+e^{-\beta c}},
$$

with $c$ being the coherence of the motion stimulus and $\beta$ being the only free parameter of the model, which determines the subject's sensitivity to the motion stimulus. This model suggests that subjects make a decision between two possible alternatives on the basis of a net evidence signal directly proportional to the motion coherence. We reasoned that in the presence of an interfering stimulus, the subjects might still be making a single decision between two alternatives, but that the net evidence signal upon which the decision is based might now be composed of two additive components: a term proportional to the motion coherence along the relevant dimension, and another term proportional to the motion coherence along the irrelevant dimension. A successful attenuation of the irrelevant component or a successful enhancement of the relevant component could be reflected by a stronger weighting of the relevant component against the irrelevant component. Thus, our model for the probability of making a correct choice in the presence of an interfering motion component has the following form:

$$
P(\text { correct })=\frac{1}{1+e^{-\left(\beta_{\text {rel }} \cdot c_{\text {rel }}+\beta_{\text {irr }} \cdot c_{\text {irr }}\right)}},
$$

in which $c_{\text {rel }}$ is the motion coherence of the relevant component (in our case, either $+10 \%$ or $+20 \%$ ) and $c_{\text {irr }}$ is

Table 1

Experimental Conditions

\begin{tabular}{lcclcccc}
\hline $\begin{array}{c}\text { Condition } \\
\text { Name }\end{array}$ & $\begin{array}{c}\text { Relevant } \\
\text { Coherence } \\
(\%)\end{array}$ & $\begin{array}{c}\text { Irrelevant } \\
\text { Coherence } \\
(\%)\end{array}$ & $\begin{array}{c}\text { Stimulus } \\
\text { Conflict }\end{array}$ & $\begin{array}{c}\text { Response } \\
\text { Targets }\end{array}$ & $\begin{array}{c}\text { Response } \\
\text { Conflict }\end{array}$ & $\begin{array}{c}\text { Mean Error } \\
\text { Rate }(\%)\end{array}$ & $\begin{array}{c}\text { Mean RT } \\
(\mathrm{sec})\end{array}$ \\
\hline conHL & 20 & 10 & low & congruent & absent & 17.1 & 1.16 \\
conHH & 20 & 20 & medium & congruent & absent & 18.3 & 1.18 \\
conLL & 10 & 10 & medium & congruent & absent & 30.1 & 1.24 \\
conLH & 10 & 20 & high & congruent & absent & 26.7 & 1.22 \\
incHL & 20 & 10 & low & incongruent & present & 30.1 & 1.26 \\
incHH & 20 & 20 & medium & incongruent & present & 38.4 & 1.26 \\
incLL & 10 & 10 & medium & incongruent & present & 40.4 & 1.26 \\
incLH & 10 & 20 & high & incongruent & present & 49.8 & 1.30 \\
\hline
\end{tabular}

Note-con, congruent; inc, incongruent; H, high; L, low. 
the motion coherence of the irrelevant component (in our case, either $+10 \%$ or $+20 \%$ for response-congruent trials or $-10 \%$ or $-20 \%$ for response-incongruent trials). Note that when $c_{\text {irr }}$ is positive, irrelevant motion is consistent with relevant motion; this should, according to the model, improve accuracy. $\beta_{\text {rel }}$ is the weight applied to the relevant component, and $\beta_{\text {irr }}$ is the weight applied to the irrelevant component. Equivalently, the error rate is given by

$$
P(\text { error })=1-P(\text { correct })=\frac{e^{-\left(\beta_{\mathrm{rel}} \cdot c_{\mathrm{rel}}+\beta_{\mathrm{ir}} \cdot c_{\mathrm{irr}}\right)}}{1+e^{-\left(\beta_{\mathrm{rel}} \cdot c_{\mathrm{rel}}+\beta_{\mathrm{irr}} \cdot c_{\mathrm{irr}}\right)}} .
$$

As we will see, this simple two-parameter model provided a convincing explanation of the pattern of error rates across our eight experimental conditions, suggesting that our subjects were indeed still making a single decision between two alternatives on the basis of a sensory evidence signal that was strongly determined by the motion along the relevant dimension and more weakly influenced by the motion along the irrelevant dimension.

Clear predictions for behavior can be derived from the model: We would expect that increased coherence of motion (relevant or irrelevant) that supports the correct response would always lead to improvements in performance, whereas increased (irrelevant) coherence of motion that competes with the correct response should lead to decrements in performance. It follows that there should be an interaction between response conflict and stimulus conflict, such that the latter has greater negative impact when the former is high. Moreover, changes in coherence on the relevant dimension should have more impact on performance than should similar changes in the irrelevant dimension.

In addition, the model, in combination with data from monkeys, suggests likely patterns of activation in areas homologous to the MT and LIP areas. Neural firing increases in the MT area as a function of motion coherence, so we would expect a similar pattern of blood oxygen level dependent (BOLD) activation in the homologous region in humans (hMT). Moreover, on the basis of evidence that attention to a particular direction increases gain on direction-selective neurons in the MT area (Treue \& Martinez Trujillo, 1999), we would expect to see greater modulation of hMT activation associated with changes in relevant (attended) motion coherence than with changes in irrelevant (unattended) motion coherence. Simultaneous accumulation of evidence in favor of both possible responses, as occurs when response conflict is present, should lead to a substantial coactivation of both subpopulations of neurons coding for the two possible responses and, therefore, an overall increased activity in the LIP area; we thus predicted increased parietal activation associated with response conflict in the present study. It is important to note, however, that the model is a model of bottom-up processing and does not incorporate mechanisms of cognitive control. Furthermore, it captures only the statistics of how, overall, subjects should distribute their choices across trials, and does not consider potential trial-by-trial variations in the model parameters (since they might be caused by fluctuations in cognitive control). We therefore took these model predictions as a starting point only and further hypothesized that the MT area-representing, as it does, the sensory information upon which the decision has to be based - could be a potential target for resolving stimulus conflict after it has been detected by a cognitive control mechanism. Likewise, the LIP area, which reflects accumulated evidence for different action alternatives, could be a potential target for resolving response conflict, once detected. We therefore hypothesized that stimulus conflict should primarily have an impact on hMT activation, whereas response conflict's impact should primarily be seen on parietal activation.

In addition to the human homologues of the MT and LIP areas, further brain regions were expected to be involved in cognitive control associated with stimulus conflict and response conflict. On the basis of consistent findings from prior studies, we expected to find activation in the dACC related to response conflict and in the DLPFC related to the imposition of top-down control. Given the varied results seen in the prior literature on stimulus conflict, the predictions here were less certain; key questions were (1) whether or not the dACC would be as engaged by stimulus conflict as it is by response conflict, and (2) which regions, if any, would be engaged specifically by stimulus conflict.

\section{METHOD}

\section{Subjects}

Fourteen healthy, right-handed young adults were included in this fMRI study ( 6 women; mean age, 22.3 years). Subjects were recruited from the University of California at Davis and were paid for their participation. Informed consent was obtained from all subjects, and all procedures were approved by the Internal Review Board at UC Davis.

\section{Procedure}

Each experimental trial proceeded as follows. First, following a brief prompt, a pair of response targets were revealed. These targets were always located at opposite corners of the screen: either top left and bottom right, or top right and bottom left. Next, a vertical or horizontal double-arrow appeared onscreen. Subjects were instructed to attend to the dimension cued by this arrow. Next, a dot-motion stimulus appeared for $1 \mathrm{sec}$. There was always some coherent horizontal motion left or right and some coherent vertical motion up or down. Subjects reported seeing separable horizontal and vertical motion with these stimuli, rather than an oblique vector average. Finally, the dot-motion stimulus disappeared, and subjects were allowed $1 \mathrm{sec}$ to complete their response. Detailed trial timing is shown in Figure 1.

Subjects held a button box in each hand, with two buttons available on each. Left-sided responses were made by pressing a button on the left-hand box, and right-sided responses were made by pressing a button on the right-hand box. Subjects were instructed to pick the target closest to the identified relevant direction of motion; for example, if the identified direction was up, the correct target would be on the top (either top left or top right). The button boxes were arranged so that button positions corresponded naturally with possible target locations (i.e., top left button with top left target); all subjects reported that the mapping was obvious and easy. To facilitate comparison with the nonhuman primate dot-motion studies, we asked subjects to make a saccade to the selected target in addition to responding via buttonpress.

Each subject was instructed on the task and completed a few sample trials before entering the fMRI scanner. Once inside the scanner, each subject practiced the task until comfortable with it, then 

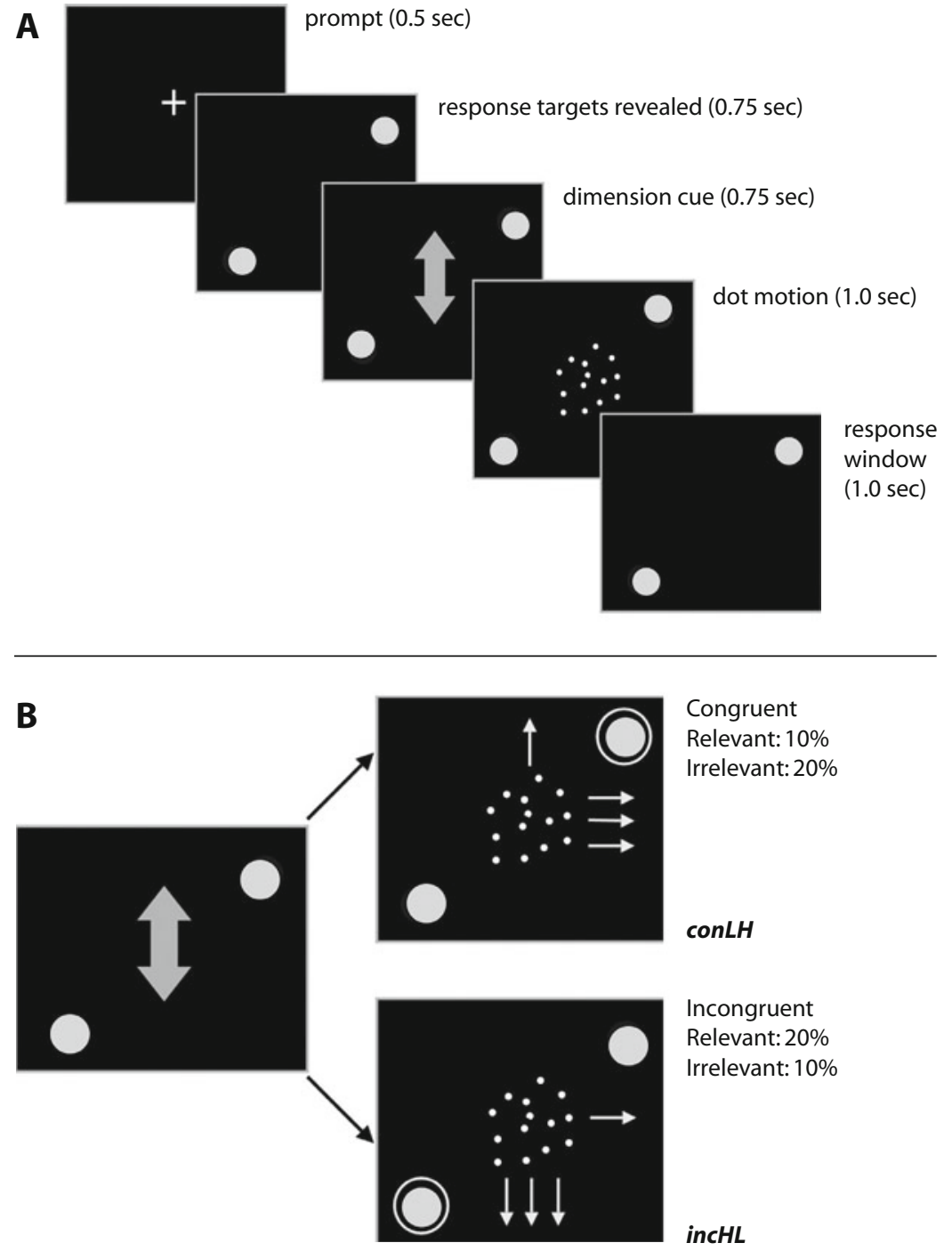

Figure 1. (A) Depiction of the sequence of events in a single trial. (B) Examples of two different trial types. The small arrows indicate direction of motion, and the number of small arrows denotes motion strength (coherence). Correct response targets are circled. These additional arrows and circles are for illustration only and were not present in the stimuli. Note that the actual display included many more dots than are shown here. con, congruent; inc, incongruent; L, low; H, high.

completed four 10-min task runs in the fMRI scanner. There were 40 repetitions of each of the eight conditions across the four 10-min runs. Each run also included 20\% null fixation for the jittered intertrial intervals (ITIs). The ordering of conditions, and the assignment of ITIs, was optimized using the program OPTSEQ2 (http://surfer .nmr.mgh.harvard.edu/optseq/).

\section{Data Acquisition}

Scanning was performed on a 3T fMRI scanner (Siemens Trio) at the University of California Imaging Research Center, using an open whole-head coil. Visual stimuli were projected onto a screen that was viewed through a mirror mounted above the fMRI head coil. Stimulus presentation and response acquisition were controlled by the Presentation software system (http://nbs.neuro-bs.com). Head motion was restricted by foam inserts that surrounded the head. Subjects wore earplugs and earphones (for communication between scans) and held a button box in each hand.
Functional data were acquired using a gradient-echo echo-planar pulse sequence (TR, $2.0 \mathrm{sec}$; TE, $28 \mathrm{msec}$; 34 axial slices, $3.44 \times$ $3.44 \times 4 \mathrm{~mm} ; 300$ volumes per run). Five extra volumes were discarded from the beginning of each run to allow for T1-equilibration effects. In addition to the functional scans, high-resolution T1-weighted (MP-RAGE) anatomical images were collected from each subject.

\section{Data Analysis}

Data were preprocessed and analyzed using SPM2 (Wellcome Department of Cognitive Neurology, London). First, functional images were corrected for differences in slice acquisition timing, and were realigned to the first volume by means of rigid body motion correction with sinc interpolation. Structural and functional images were spatially normalized to T1 and EPI templates, respectively. These templates are based on the MNI stereotaxic space (Cocosco, Kallokian, Kwan, \& Evans, 1997), an approximation of the Talairach 
space (Talairach \& Tournoux, 1988). The normalization algorithm involved a 12-parameter affine transformation, together with a nonlinear transformation involving cosine basis functions. During normalization, the volumes were resampled to $3 \times 3 \times 3 \mathrm{~mm}$ cubic voxels. Functional images were spatially smoothed with an $8-\mathrm{mm}$ full-width half-maximum isotropic Gaussian kernel. The smoothed, normalized images were then submitted to statistical analyses.

Our primary analysis involved consideration of four factors: response conflict, relevant coherence, irrelevant coherence, and correctness of response. A general linear model (GLM) incorporating task effects, session effects, and a linear trend was used to analyze each subject's data. For each subject, this model was used to compute the least-squares parameter estimate of the height of the best-fitting synthetic response function for each condition at each voxel. Task-related neural activity was modeled as a series of zero-duration events aligned to the onsets of the dot-motion stimuli; this series was then convolved with SPM's canonical hemodynamic response function (HRF) to produce a model of the predicted BOLD response. High-pass temporal filtering with an upper limit of $120 \mathrm{sec}$ was applied to eliminate low-frequency noise from the data. Contrast images were computed within subjects for each comparison of interest. Random-effects analyses were performed on the individual subject's contrast images to generate group contrast maps. Except where otherwise noted, group maps were thresholded using an uncorrected $p$ value of .001 and an extent threshold of five contiguous voxels.

For select activation clusters derived from the whole-brain contrasts, region-of-interest (ROI) analyses were performed. This secondary analysis allowed for characterization of a region in terms of its activation across multiple experimental factors. ROIs were constructed and analyzed with the Marsbar toolbox (Brett, Anton, Valabregue, \& Poline, 2002; http://marsbar.sourceforge.net). Mean contrast values for each subject and condition were extracted for each ROI and submitted to multifactorial ANOVAs.

\section{Model Fit}

Behavioral error rates were fit to a mathematical model (see above). For fitting the model, all individual responses from all subjects were treated as a single data pool, and the two model parameters $\left(\beta_{\text {rel }}\right.$ and $\left.\beta_{\text {irr }}\right)$ were determined using maximum likelihood estimation.

\section{RESULTS}

\section{Behavioral Performance}

In general, increases in both stimulus conflict and response conflict tended to impair task performance (see Figure 2; solid bars). Response-congruent trials were performed more quickly $[F(1,12)=38.2, p<.0001]$ and more accurately $[F(1,13)=94.7, p<.0001]$ than were response-incongruent trials. Increased motion coherence in the relevant dimension significantly aided performance [accuracy, $F(1,13)=39.9, p<.0001 ; \mathrm{RT}, F(1,13)=12.9$, $p<.001]$, whereas, overall, increased motion coherence on the irrelevant dimension slightly degraded it [accuracy: $F(1,13)=5.2, p<.05]$. However, there was an interaction between response congruence and the level of coherence in the irrelevant dimension, such that increased irrelevant coherence impaired accuracy on incongruent trials but not on congruent trials $[F(1,13)=10.5, p=.006]$. This is easily understood as a consequence of the fact that on congruent trials, additional evidence along the irrelevant dimension is still evidence that supports the correct response, and it is consistent with our mathematical model of the task (see below). Contrary to expectations, the presence of neither stimulus conflict nor response conflict on one trial had a significant effect on performance of the subsequent trial. In addition, there was no significant subsequent-trial effect of error.

\section{Mathematical Model of the Error Rates}

When our mathematical model was fit to the behavioral data, the two model parameters were determined to be $5.60\left(\beta_{\text {rel }}\right)$ and $2.72\left(\beta_{\text {irr }}\right)$, respectively. This suggests that, on average, the relevant motion signal had approximately twice as much impact on the subjects' choice as the irrelevant motion signal. The dotted bars in Figure 2 show the model error rates, and a comparison with the solid bars

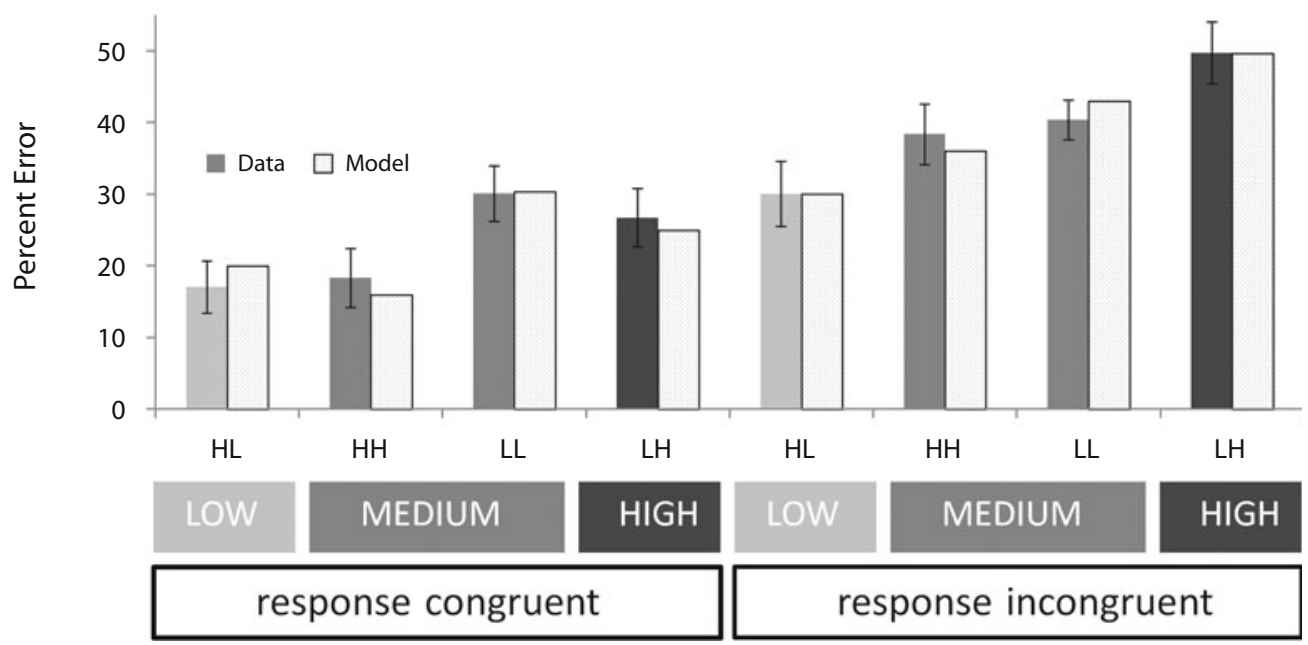

Figure 2. Behavior performance (error as a percentage) and model results (solid bars and dotted bars, respectively). Labels on the horizontal axis indicate levels of motion coherence in the relevant (first) and irrelevant (second) dimensions: H, high; L, low. Additional labels indicate the associated levels of stimulus conflict (low, medium, or high) and the response conflict status (response congruent or response incongruent). Error bars show the standard errors of the means. 

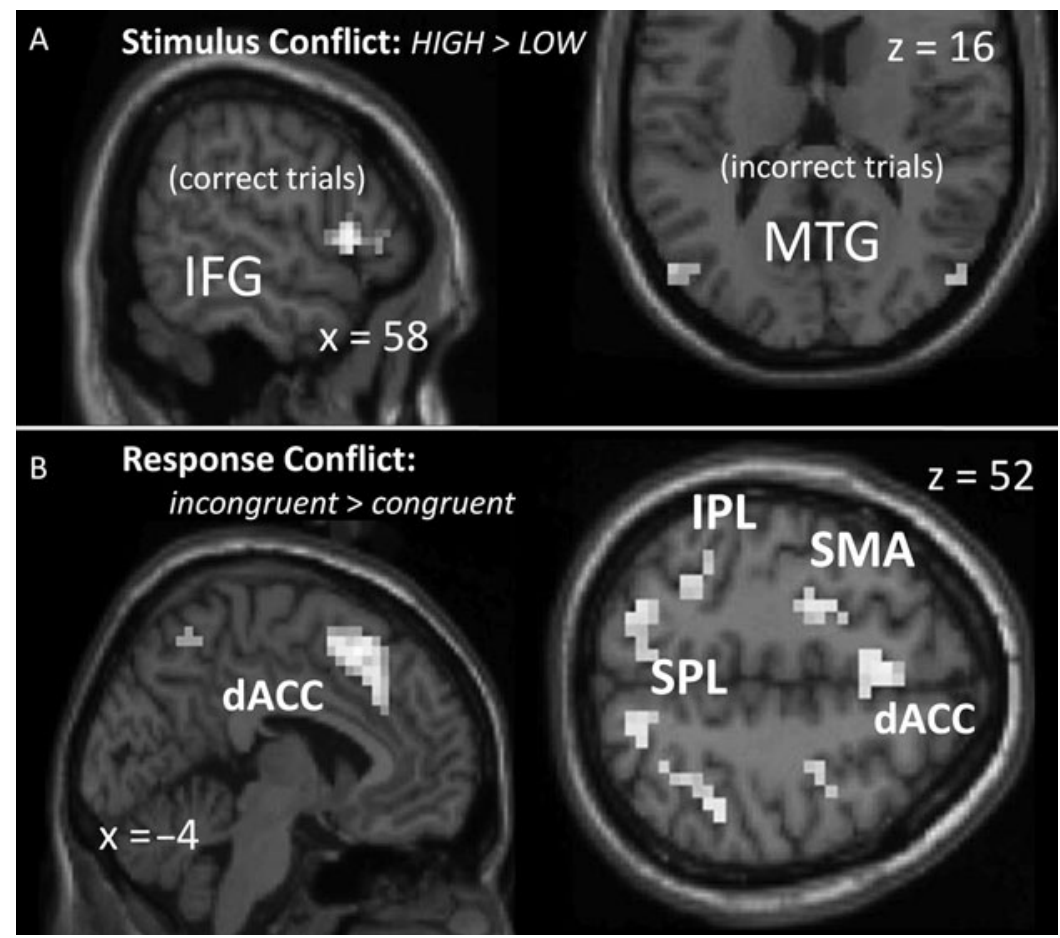

Figure 3. (A) Regions of activation associated with the contrast between trials with high stimulus conflict and trials with low stimulus conflict, for correct trials only (left) and for incorrect trials only (right). The bilateral middle temporal gyrus (MTG) was activated first when all trials were considered, then when only incorrect trials were. When only correct trials were considered, the right inferior frontal gyrus (IFG) was the only significant activation $(p<.001,>5$ voxels). (B) Activation clusters associated with response conflict, obtained from the contrast between correct incongruent and correct congruent trials. The dorsal anterior cingulate cortex (dACC), supplementary motor area (SMA), and parietal cortex, including the superior parietal lobe (SPL) and inferior parietal lobe (IPL), were activated by this contrast $(p<.001,>5$ voxels).

next to them (data) indicates that the pattern of error rates across all eight experimental conditions was well captured by our simple two-parameter model.

\section{Stimulus Conflict}

Stimulus conflict was first probed via examination of the contrast between trials for which irrelevant coherence exceeded relevant coherence (LH; high stimulus conflict) and those that had the opposite pattern (HL; low stimulus conflict), since this was the contrast for which we expected the stimulus conflict effect to be most evident. Note that this contrast excluded trials that involved equivalent levels of motion coherence along the relevant and irrelevant dimensions (HH, LL; medium stimulus conflict). For correct trials, this stimulus conflict contrast revealed a locus of activation in the right IFG $(52,20,-4)$; no other regions were significantly activated (Figure 3A, left; Table 2). Because correct performance is an indicator of successful cognitive control and incorrect performance is an indicator of a lack of control, we were motivated to examine incorrect trials as well as correct trials. When the stimulus conflict contrast was limited to incorrect trials, activation was instead observed at the junction of the middle temporal and occipital gyri (MTG/MOG), bilaterally $(-48,-76,4$ and $52,-76,12$; Figure $3 \mathrm{~A}$, right, and Table 2). These bilateral
MTG/MOG activation clusters are consistent with the hMT area, the likely human homologue of the monkey MT area (Huk, Dougherty, \& Heeger, 2002; Watson et al., 1993).

Our experimental design also afforded separate investigation of the effects of relevant and irrelevant percept strength. Because stimulus conflict increases with increasing motion coherence in the relevant dimension and also with decreasing motion coherence in the relevant dimension, we examined two contrasts: high irrelevant coherence $>$ low irrelevant coherence $(\mathrm{HH}+\mathrm{LH}>\mathrm{HL}+\mathrm{LL}$; any effect due only to changes in the relevant coherence should disappear) and low relevant coherence $>$ high relevant coherence $(\mathrm{LH}+\mathrm{LL}>\mathrm{HH}+\mathrm{HL}$; any effect due only to changes in the irrelevant coherence should disappear). Both contrasts, taken across correct trials, revealed activation only in the right IFG (Table 2); the contrast between low- and high-relevant coherence revealed a cluster that largely overlapped with the previous IFG activation cluster, whereas the contrast between low- and high-irrelevant coherence revealed IFG activation medial to these other clusters. Taken across incorrect trials only, neither contrast revealed any significant areas of activation, although the right MTG/MOG was activated by the manipulation of irrelevant motion coherence at a relaxed threshold of $p<.005$. 
Table 2

Areas of Activation for Selected Contrasts

\begin{tabular}{|c|c|c|c|c|}
\hline Region & $x, y, z$ & $T$ & $\begin{array}{c}\text { Cluster } \\
\text { Size }\end{array}$ & $P$ (Cluster) \\
\hline \multicolumn{5}{|c|}{ Stimulus Conflict } \\
\hline \multicolumn{5}{|c|}{ Stimulus conflict: High $>$ Low (LH $>$ HL, correct trials) } \\
\hline Right IFG (BA 45, 47) & $60,15,3$ & 7.69 & 25 & .025 \\
\hline \multicolumn{5}{|c|}{ Irrelevant coherence: High $>$ Low $(* \mathrm{H}>* \mathrm{~L}$, correct trials $)$} \\
\hline Right IFG (BA 47) & $33,24,-12$ & 4.67 & 10 & .18 \\
\hline \multicolumn{5}{|c|}{ Relevant coherence: Low $>$ High $\left(\mathrm{L}^{*}>\mathrm{H}^{*}\right.$, correct trials) } \\
\hline Right IFG (BA 45, 47) & $60,15,3$ & 8.16 & 25 & .018 \\
\hline \multicolumn{5}{|c|}{ Stimulus conflict: High $>$ Low $(\mathrm{LH}>\mathrm{HL}$, incorrect trials) } \\
\hline Left MOG/MTG & $-48,-76,4$ & 5.5 & 19 & .09 \\
\hline Right MTG/MOG & $52,-76,12$ & 6.72 & 40 & .02 \\
\hline \multicolumn{5}{|c|}{ Response Conflict } \\
\hline \multicolumn{5}{|c|}{ Incongruent $>$ Congruent (correct trials) } \\
\hline dACC (BA 6, 8, 32) & $-4,28,44$ & 5.25 & 58 & .001 \\
\hline Precentral gyrus (BA 6) & $-36,0,32$ & 5.02 & 36 & .005 \\
\hline Left MFG (BA 6) & $-24,-8,56$ & 4.30 & 19 & .029 \\
\hline Right MFG (BA 6) & $28,-8,56$ & 4.26 & 24 & .016 \\
\hline Left IPL (BA 40) & $-32,-52,52$ & 3.91 & 14 & .056 \\
\hline Right IPL (BA 40) & $40,-40,48$ & 4.56 & 26 & .013 \\
\hline Left SPL (BA 7) & $-24,-72,56$ & 4.36 & 23 & .018 \\
\hline Right SPL (BA 7) & $16,-68,52$ & 4.09 & 9 & .117 \\
\hline Right MTG (BA 37) & $48,-68,0$ & 4.68 & 10 & .100 \\
\hline Left MOG (BA 18) & $-28,-96,12$ & 6.04 & 69 & $<.001$ \\
\hline Right MOG (BA 18) & $16,-96,0$ & 4.95 & 55 & .001 \\
\hline
\end{tabular}

Note-Areas of activation are at a voxelwise threshold of $p<.001$ (uncorrected) with an extent threshold of five voxels. IFG, inferior frontal gyrus; dACC, dorsal anterior cingulate cortex; MOG, middle occipital gyrus; MTG, middle temporal gyrus; MFG, middle frontal gyrus; IPL, inferior parietal lobe; SPL, superior parietal lobe.

ROI analyses were conducted to further examine the pattern of activation in each of these IFG and MTG/MOG clusters. Data from each region were submitted to three separate ANOVAs: a four-way $(2 \times 2 \times 2 \times 2)$ ANOVA that included trial accuracy, response conflict, relevant coherence, and irrelevant coherence; a three-way $(2 \times 2 \times 2)$ ANOVA that included trial accuracy, response conflict, and stimulus conflict; and a three-way $(2 \times 2 \times 2)$ ANOVA that included trial accuracy, response conflict, and mean motion coherence. Note that only low and high stimulus conflict conditions (HL and $\mathrm{LH}$ ) were included in the first threeway ANOVA, and only medium stimulus conflict conditions ( $\mathrm{LL}$ and $\mathrm{HH}$ ) were included in the second. Because of the small number of trials that fell in some cells of these ANOVAs - the smallest cell (conHL error) had an average of only seven trials per subject - we will consider only independent main effects and first-order interactions.

In the right IFG cluster, which was obtained from the first stimulus conflict contrast ( $\mathrm{LH}>\mathrm{HL}$, correct trials only), the strongest correct-trial activation was seen when stimulus conflict was highest (Figure 4A). There was neither an interaction between relevant or irrelevant coherence and accuracy nor an interaction between relevant or irrelevant coherence and response conflict (all $F_{\mathrm{s}}<1$ ). Similarly, there was no interaction between stimulus conflict and trial accuracy $(F<1)$, nor was there a significant interaction between stimulus conflict and response conflict $[F(1,13)=1.5, p=.25]$. There was also no effect of mean coherence $(F<1)$.
Within the MTG/MOG cluster (obtained from the LH $>$ HL contrast across incorrect trials), there was a significant interaction between trial accuracy and stimulus conflict $[F(1,13)=7.2, p=.018]$, such that, although there was a trend toward increased activation for increased stimulus conflict across correct trials, the effect of stimulus conflict was greater for incorrect trials (Figure 4B). In fact, MTG/MOG activation was greater for correct trials than for incorrect trials when stimulus conflict was low, but greater for incorrect trials than for correct trials when stimulus conflict was high. In the four-way ANOVA, a similar interaction was observed between accuracy and relevant coherence $[F(1,13)=5.4, p=.038]$ : Increased relevant coherence had a positive effect on activation on correct trials, but a negative effect on incorrect trials. Consistent with the exploratory analysis, the stimulus conflict effect in the MTG/MOG was driven largely by increased activation for trials with high coherence on the irrelevant dimension, since the main effect of irrelevant coherence was stronger than that of relevant coherence $[F(1,13)=$ 14.1 vs. $F(1,13)=1.78$; but note that these effects were not independent of the contrast used to select the ROI]. There was a trend toward increased MTG/MOG activation associated with increased mean coherence, although this was not significant $[F(1,13)=2.1, p=.17]$.

In summary, the right IFG was engaged by stimulus conflict, but only on correct trials, whereas the bilateral MTG/ MOG was engaged by stimulus conflict preferentially on incorrect trials. IFG activation was strongly affected by 


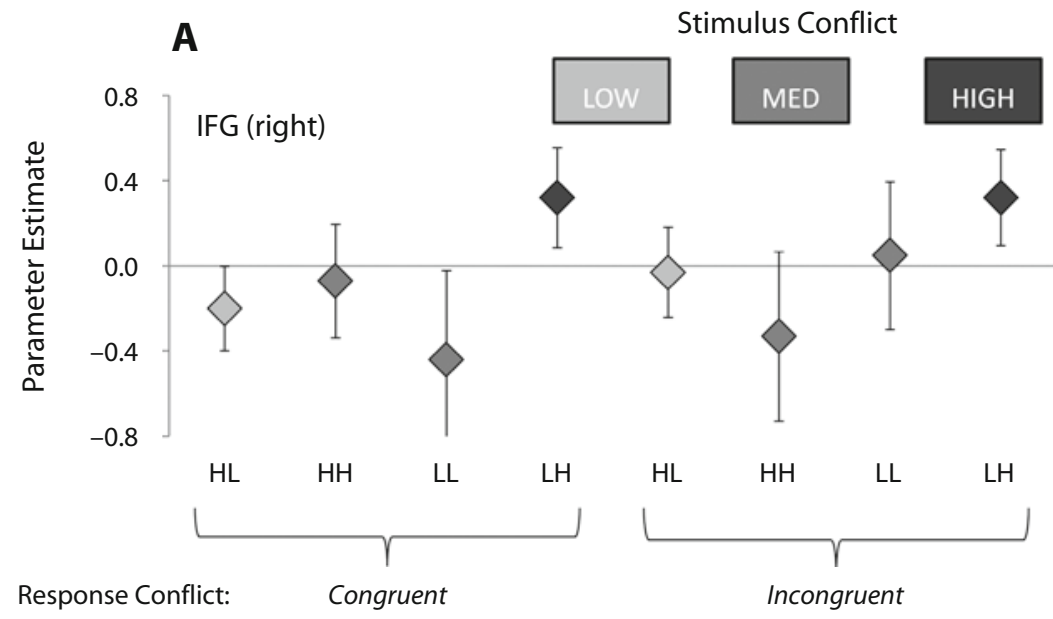

B

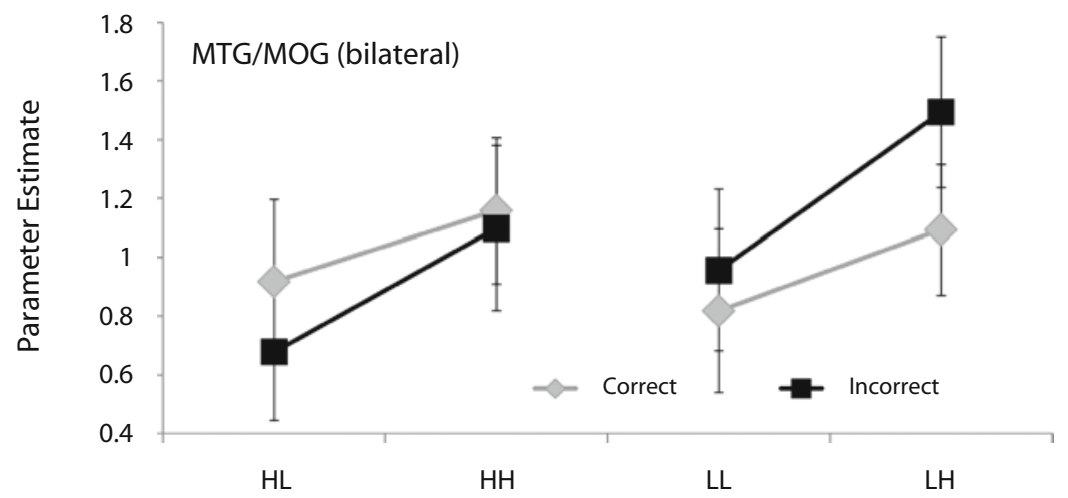

Figure 4. Activation pattern in functional regions of interest associated with stimulus conflict. (A) Parameter estimates across all correct trials for the right inferior frontal gyrus (IFG). This region was obtained from the LH-HL (high-low) stimulus conflict contrast, limited to correct trials. (B) Activation in the bilateral middle temporal and occipital gyri (MTG/MOG), showing the effect of motion coherence in the irrelevant dimension, especially for trials that were responded to incorrectly. The MTG/MOG region was obtained from the LH-HL (high-low) stimulus conflict contrast, limited to incorrect trials.

changes to both relevant and irrelevant motion coherence. The MTG/MOG, on the other hand, was affected mainly by changes in irrelevant motion coherence.

\section{Response Conflict}

Response conflict was probed via examination of the contrast between response-incongruent and responsecongruent correct trials. Several regions were activated by this contrast, including, most prominently, the dACC $(-4,28,44$; including the ACC and the adjoining area of the posterior medial frontal gyrus), the bilateral supplemental motor area (SMA), and the bilateral SPL and IPL (Figure 3B).

ROI analyses were conducted to examine the pattern of activation across conditions for each of these activation clusters. For the dACC cluster obtained from the contrast between response-incongruent and response-congruent correct trials, each response-incongruent condition was associated with greater activation than was every response- congruent condition, regardless of coherence levels (Figure $5 \mathrm{~A})$. In a three-way $(2 \times 2 \times 2)$ ANOVA that included trial accuracy (correct vs. incorrect), response conflict, and stimulus conflict as factors, there was a significant main effect of accuracy $[F(1,13)=13.8, p=.003]$; in effect, incorrect trials were similar to correct incongruent trials in terms of dACC activation. There was no main effect of stimulus conflict in the dACC, nor was there an interaction between stimulus conflict and response conflict $(F \mathrm{~s}<1)$.

The SMA, SPL, and IPL all showed a pattern similar to that of the dACC - namely, increased activation for incorrect as well as for incongruent trials, but no main effect of stimulus conflict. In a secondary analysis, response laterality was included as a factor in the GLM, and separate correct-trial data from the left and right SMA, SPL, and IPL were submitted to $2 \times 2 \times 2$ ANOVAs that included response laterality and brain side as well as response conflict as factors. Response laterality proved to be a signifi- 

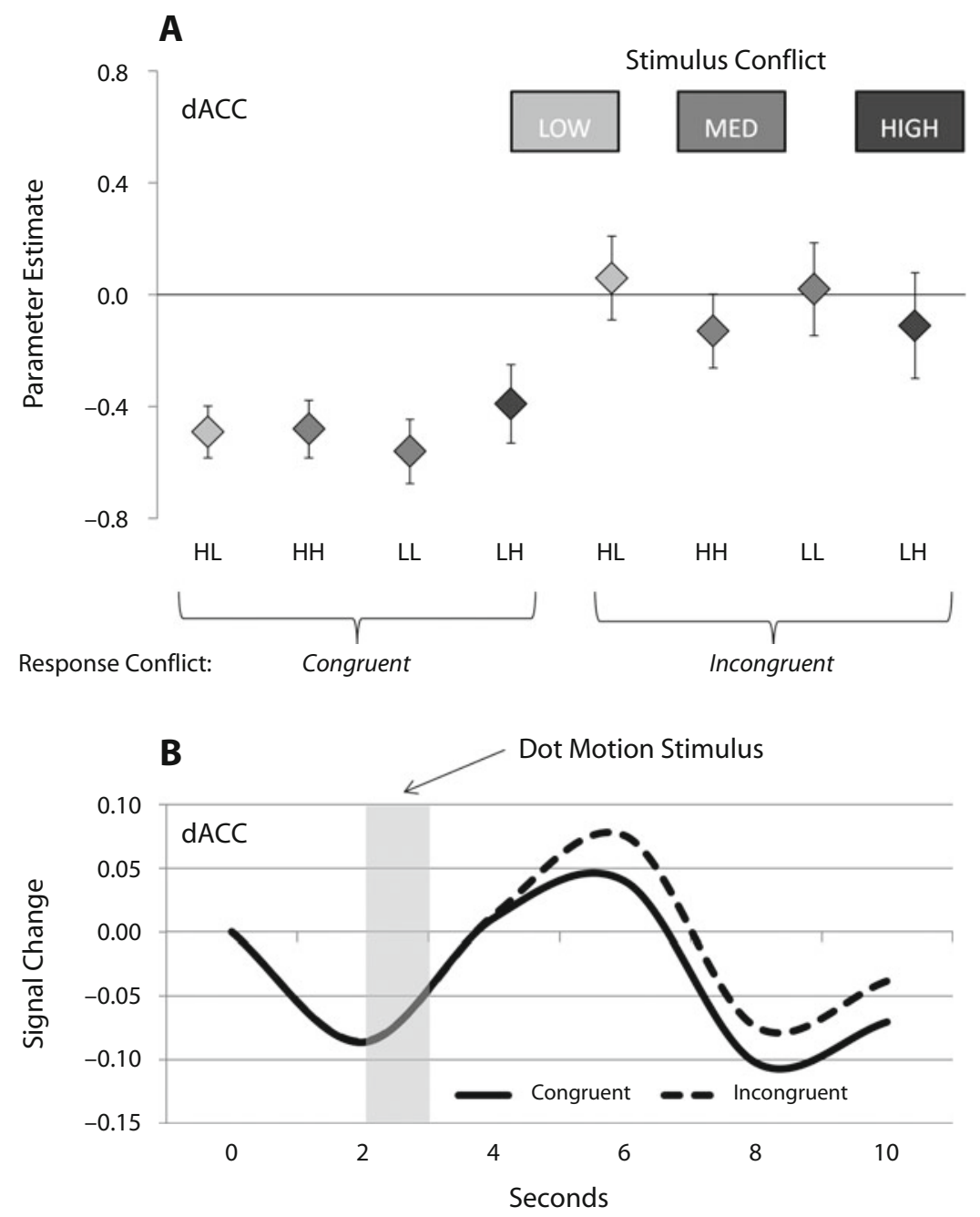

Figure 5. Data from the dorsal anterior cingulate cortex (dACC) region of interest, which was obtained from the incongruent-congruent response conflict contrast. (A) Parameter estimates across all correct trials. (B) BOLD activation time course, using signal value at trial onset as a baseline. The shaded area depicts the onset and duration of the dot motion stimulus.

cant determinant of SMA activation. Specifically, there was a significant interaction between the SMA side and response laterality $[F(1,13)=15.9, p<.001]$, such that the SMA region contralateral to the response was more highly activated than the ipsilateral region was. Response laterality was not, however, a factor in the parietal regions.

The difference in parameter estimates between incongruent and congruent trials in the dACC manifested itself as a decrease from zero in the congruent condition, rather than as a significant increase from zero in the incongruent condition. Examination of the congruent and incongruent trial time courses for the dACC sheds light on this, revealing a large initial decrease in BOLD for both trial types, followed by an increase (corresponding to the dot-motion stimulus) larger for incongruent than for congruent trials (Figure 5B). Our parameter estimates, clearly, were affected by the large early-trial deactivation, as well as by the differential activation that occurred in response to the dot-motion stimulus.

\section{Sequential Effects}

Although there were no significant behavioral effects of previous-trial conflict, we speculated that this was due to the overall difficulty of the task and that brain effects might still be detectable. Therefore, two additional analyses classified trials on the basis of (1) the level of stimulus conflict and (2) the level of response conflict on the previous trial. In the contrast between high and low levels of previous-trial stimulus conflict, no regions were activated at the a priori threshold. However, at $p<.005$ (uncorrected), the right DLPFC (BA 9; 57, 18, 36) appeared as the only locus of activation for previous-trial stimulus conflict. Similarly, the right DLPFC (BA 9/46; 57, 24, 30) was the region most prominently activated by previoustrial response conflict, although in this case the activation in this region was detected only at $p<.01$ (uncorrected). However, DLPFC activation was not observed on trials following errors, even at $p<.01$ (uncorrected). In sum- 
mary, the right DLPFC was the region most strongly associated with previous-trial conflict, although the strength of that association was only marginally significant.

\section{DISCUSSION}

Results from the present study allow us to clearly disambiguate stimulus conflict from response conflict, showing that different brain regions are engaged in the presence of the former versus the latter. In particular, it is shown that the dACC is engaged by response conflict, but the right IFG is engaged by stimulus conflict. Moreover, the present results complement prior findings from the study of perceptual decision making in monkeys, which has focused on the role of MT and LIP areas, by providing insight into the specific functions of homologous human areas in the MTG/MOG and the parietal cortex during perceptual decision making in the presence of conflict.

\section{Stimulus Conflict}

Stimulus conflict occurs whenever there is irrelevant perceptual information that competes for attentional resources with goal-relevant perceptual information. Most instances of perception involve some degree of stimulus conflict, but conflict is greater when irrelevant percepts are more salient than relevant percepts.

Activation in the MTG/MOG reflected the amount of stimulus conflict. This modulation of MTG/MOG activation by stimulus conflict was most evident on trials where subjects responded incorrectly. All else being equal, an error indicates a shift of attention toward the irrelevant dimension, most probably manifested as an increase in gain applied to the irrelevant dimension and/or a decrease in gain applied to the relevant dimension. This fact yields two key predictions for a region that encodes motion direction and contributes to the decision-making process. First, when relevant motion coherence is stronger than irrelevant motion coherence, error trials will be associated with relative attenuation of the stronger signal and relative amplification of the weaker signal. Second, when irrelevant motion coherence is stronger than relevant motion coherence, error trials will be associated with relative amplification of the stronger signal and attenuation of the weaker signal. Thus, if the MTG/MOG region identified here encodes motion and supports decision making, it should show greater activation on error trials than on correct trials when stimulus conflict is high and greater activation on correct trials than on error trials when stimulus conflict is low. This particular interaction between accuracy and stimulus conflict is exactly what was observed. It should be noted, however, that error trials are associated with many things - arousal, error detection, and response conflict, to name a few-so our interpretation of this interaction is by no means definitive.

The MTG/MOG clusters identified here are consistent with the hMT area, which has been identified as the human equivalent of the monkey MT area (Huk et al., 2002; Watson et al., 1993). Thus, it is likely that this activated region encodes the directions and strengths of dot motion in the present study. Previous human fMRI studies have demonstrated that the hMT area, in addition to encoding motion, is subject to attentional modulation (Beauchamp, Cox, \& DeYoe, 1997). And indeed, attentional modulation of the hMT area is probably seen here in the interaction between trial accuracy and relevant coherence: The more successful deployment of attentional control on correct trials relative to incorrect trials is associated with increased gain for the target direction.

For a region involved in the top-down resolution of stimulus conflict, rather than simply in the processing of interfering percepts, activation increases associated with increasing levels of conflict should be most clearly observable on correctly performed trials, since it is on correctly performed high-conflict trials that the engagement of conflict resolution mechanisms is most likely to have occurred. The right IFG alone showed this pattern, with increased activation for high levels of stimulus conflict on correct trials but no effect of stimulus conflict on incorrect trials. Thus, it appears that the right IFG supports correct performance on trials where stimulus conflict is high. It may do so, directly or indirectly, by causing inhibition of the irrelevant perceptual signals, selective enhancement of the relevant signal, or both. Prior evidence suggests that inhibition may be a key function of the right IFG (Aron, Robbins, \& Poldrack, 2004). That the IFG was not activated for trials with medium, relative to low, stimulus conflict suggests that there may be a threshold effect, such that to engage frontal control, stimulus conflict must be sufficiently high.

The present result is most similar to that reported by van Veen et al. (2001) and by Nelson, Reuter-Lorenz, Sylvester, Jonides, and Smith (2003). The former study (van Veen et al., 2001) involved a letter flanker task in which multiple letters mapped to each response option, and they observed activation limited to the IFG (although left rather than right) when subjects had to respond to distinct target and flanker letters that mapped onto the same response. The laterality difference between the two results may be due to the different nature of the stimuli in the two tasks. The latter study (Nelson et al., 2003) compared response-based conflict with familiarity-based conflict in a Sternberg working memory task. Familiarity-based conflict was deemed to be present when a probe item was present in the previous trial, and this yielded activation in the left IFG. Response-based conflict was present when the current probe would have had an opposite response on the previous trial, and this yielded activation in the dACC. Other studies of stimulus conflict have reported activation in the PFC, although not specifically in the IFG (Liston et al., 2006; Milham et al., 2001; Weissman et al., 2003).

In addition, several studies have reported activation in the dACC and the parietal cortex related to stimulus conflict (Liston et al., 2006; Liu et al., 2004; van Veen \& Carter, 2005; Weissman et al., 2003), a pattern that corresponds better to response conflict in the present results. As we argued in the introduction, the use of learned response rules in many of these studies may have led to errors in rule lookup and, thus, to the inadvertent inclusion of response conflict in stimulus conflict trials. This possibility may in particular explain previous linkage between stimulus conflict and the dACC. By contrast, in the present study, dot 
motion maps naturally onto a target location, without the need to recall a mapping rule. Thus, the present results are less likely to be affected by rule access and should present a purer picture of activation related to stimulus conflict. Here, we did not observe any effect of stimulus conflict in either the dACC or the parietal cortex.

With regard to the investigation of stimulus conflict, a second advantage of the present study is that it allowed for examination of the separate contributions of relevant and irrelevant stimulus strength. That the IFG was engaged by motion coherence increases (irrelevant motion) as well as by decreases (relevant motion) indicates that this region is indeed modulated by stimulus conflict - or more broadly, stimulus difficulty - rather than simply by perceptual strength in either the attended or the unattended dimension. This responsivity to the higher level construct (i.e., stimulus conflict) is characteristic of a high-level control mechanism. The situation in the IFG contrasts with that in the hMT area, where the stimulus conflict effect appears to have been driven largely by increases in irrelevant motion coherence, but not by decreases in relevant motion coherence.

\section{Response Conflict}

Response conflict occurs when evidence supports multiple competing responses. In the present study, response conflict was present on incongruent trials, in which the two dimensions of dot motion corresponded to two different response options, but was not present on congruent trials, in which the two dimensions of motion corresponded to a single button response. That the dACC was the most prominent locus of activation in the contrast between incongruent and congruent trials is not surprising: Activation of this region in situations with high response conflict has been demonstrated in many different studies (Carter et al., 1998; Kerns et al., 2004; MacDonald et al., 2000). This region is also typically activated by response errors, even without overt response conflict, and that pattern was repeated here. Even where there is no externally driven response conflict, the commission of an error indicates that there was conflict induced by competition between the correct response and the chosen response. In addition to its description as a region specialized for conflict detection, the dACC region has also been described as a region specialized for error-likelihood detection (Brown \& Braver, 2007) or, more generally, for performance monitoring (Ridderinkhof, Ullsperger, Crone, \& Nieuwenhuis, 2004). Because trials with high stimulus conflict are more associated with impaired performance than are trials with low stimulus conflict, these alternate hypotheses would predict that dACC activation increases for the former relative to the latter. That this was not observed suggests that the dACC is not performing error-likelihood detection in this task, and that its possible role as a general performance monitor does not extend to the monitoring of stimulus conflict.

It is notable that, for correct incongruent trials, dACC activation did not increase as a function of increasing motion coherence for the unselected response. This is surprising, since in most models of response conflict de- tection, increased evidence for the unselected response would lead to increased response conflict. ${ }^{1}$ The observed pattern suggests that the putative stimulus conflict resolution mechanism might be modulating the inputs to the dACC. However, in this case, for incongruent trials with high stimulus conflict, we might have expected to see error rates reduced, relative to the predictions of the mathematical model. Since we did not observe this pattern in the behavior, this finding cannot be fully explained. Importantly, however, if we consider incorrect incongruent trials rather than correct incongruent trials, there was an effect in the expected direction-namely, increased coherence associated with the unselected response led to increased activation of the dACC $[F(1,13)=23.2, p<.001]$.

The observed activation of the bilateral SMA for response conflict can be explained in terms of a substantial coactivation of neural representations of both possible responses. Early stages in the motor planning pathway should show increased activation as multiple possible responses are considered as being appropriate, but this increase should diminish for later stages as, and to the extent that, conflict is resolved. The SMA is involved in the early processing of motor output (Lee, Chang, \& Roh, 1999). Thus, we would clearly expect that this region should show an effect of response conflict. Lateralization of SMA activation, such that the SMA region contralateral to the response side showed increased activation relative to the ipsilateral region, reinforces the conclusion that this region is involved in motor planning and, in particular, that it is involved at a later stage than the SPL, which did not show the same pattern of lateralization.

Activation related to response conflict in the SPL, bilaterally, suggests that this region is also involved in the processing of response options. Recent fMRI studies comparing monkey and human fMRI have suggested that a region within the SPL near the medial wall of the intraparietal sulcus corresponds to the monkey LIP (Grefkes \& Fink, 2005). SPL activation in the present study appears to include the medial wall of the intraparietal sulcus; therefore, it is likely that this region, or at least part of it, corresponds to the LIP area identified in monkeys (Shadlen \& Newsome, 2001). The LIP area was shown to be a locus of evidence accumulation and a place where visual inputs are mapped to motor outputs. A region with this functionality would be expected to show increased activity during situations with high response conflict, since the presence of conflict would correspond to strong activation within multiple subpopulations of neurons corresponding to competing response options. That there was no effect of response laterality in the SPL indicates that the accumulation of evidence for a lateralized response seems to occur across both hemispheres in humans. The lack of SPL lateralization seen here is consistent with the results of another study that examined lateralization of activity for both manual and eye movement responses (Macaluso, Frith, \& Driver, 2007).

\section{Conflict Detection and Top-Down Control}

Previous studies have clarified the distinction between conflict detection and implementation of control. Spe- 
cifically, it has been shown that the detection of response conflict by the dACC can lead to top-down control via subsequent activation of the DLPFC and, thereby, to improvements in performance. This combined action of the dACC and the DLPFC has been demonstrated by looking at changes in performance and brain activation on one trial that depend on response congruence in the previous trial (Kerns et al., 2004). Similar trial-to-trial effects on performance have been shown for stimulus conflict (Verbruggen et al., 2006).

In the present study, we looked for but did not find significant trial-to-trial adjustments in behavior. We speculate that the failure to replicate previously identified behavioral patterns here is due to the difficulty of the present task. In most of the studies of conflict that have yielded trial-to-trial adjustments in performance, the basic task has been very easy, leaving ample room for an increase in mental resources devoted to the task when called for by the presence of conflict. By contrast, even the easiest trials in the present study required considerable focus on the part of the subject (as indicated by the fact that performance was well below $100 \%$ on even the easiest trial types). It is thus likely that subjects performing our task were always in a state of high cognitive control and were not in a position to increase this enough, in response to conflict on a single trial, to have a significant impact on performance.

Despite the failure to observe behavioral adjustments following conflict trials, we did observe marginally significant activation in the DLPFC following trials with high stimulus conflict and high response conflict. Although the level of DLPFC activation was not highly significant in either case, the right DLPFC was the most prominent locus of activation in both. Moreover, the fact that neither the dACC nor the IFG was activated by previous-trial conflict suggests that both of these regions are specialized more for the detection of conflict, or early stages of control, than for the application of top-down control that follows conflict detection.

\section{Attention and Control}

Cognitive control, as implemented by the joint operation of the dACC or the IFG and the DLPFC, supports the flexible allocation of attention to where it is needed. Two useful distinctions can be made to better characterize the role of the different brain regions and of the different attentional mechanisms. First, the regions involved can be characterized as either generators or targets of attention (Posner \& Dehaene, 1994). Second, the attentional mechanisms can be characterized as either perceptual or postperceptual (Luck \& Ford, 1998). In the present task, a perceptual attention mechanism is probably seen in the increased attention to a relevant dimension of dot motion that aids stimulus identification in the context of stimulus conflict. The present evidence suggests that the IFG plays a key role in this process, although the DLPFC may also be involved as a generator of the top-down attention signal directed toward the motion processing in the hMT. A postperceptual attention mechanism is observed in the increased attention given to stimulus-response mapping that aids response selection in the context of response conflict. Although the dACC appears to detect response conflict, it is again more likely, on the basis of the present evidence as well as on other accounts, that the DLPFC is the generator of the attention signal. Stimulus-response mappings in the parietal cortex may be a target of this postperceptual attention signal.

On the basis of the investigation of the monkey LIP area, we have postulated that the activation of the SPL reflects, at least to some extent, the process of stimulusresponse mapping. However, there is ample evidence that the SPL and other parts of the parietal cortex are directly involved in attentional processes, and this could also explain some of the activation patterns that we have observed. The SPL has been associated with both attention to features and switches in spatial attention (Corbetta, Shulman, Miezin, \& Petersen, 1995). In the present task, close attention to a specific region of space is necessary for successful performance. However, it is attention to a feature of the stimulus - motion along a particular axisthat is most important when conflict is high. It is possible that the SPL activation associated with response conflict reflects, at least in part, the generation of a feature-based attention signal; however, if the SPL primarily subserved feature-based attention, one would expect it to be most activated by stimulus conflict, when feature-based attention is needed the most, rather than by response conflict, as was observed.

\section{Summary}

The present study demonstrates that distinct brain regions are engaged in the presence of stimulus conflict and response conflict. In line with previous studies, the dACC was shown to be sensitive to response conflict. On the other hand, the right IFG was engaged by stimulus conflict. In addition, two brain regions previously linked to perceptual decision making were differentially engaged by stimulus and response conflict during our perceptual decision-making task. The hMT area, previously linked to processing of the motion percept, was strongly affected by the presence of stimulus conflict; the SPL, on the other hand, which has been associated with accumulation of evidence for a response, was affected by the presence of response conflict. These results shed light on the neural mechanisms that support decision making in the presence of conflict, a cognitive operation that is fundamental to both basic survival and high-level cognition.

\section{AUTHOR NOTE}

This work was supported by NIH Grant R01MH66629 to C.S.C. and by startup funds from University of California at Davis to S.A.B. We thank John Ryan and Mike Buonocore for their assistance at the UC Davis Imaging Research Center. Correspondence concerning this article should be addressed to C. Wendelken, 134 Barker Hall, University of California, Berkeley, CA 94720 (e-mail: cwendelken@berkeley.edu).

\section{REFERENCES}

Aron, A. R., Robbins, T. W., \& Poldrack, R. A. (2004). Inhibition and the right inferior frontal cortex. Trends in Cognitive Sciences, $\mathbf{8}$, 170-177.

Beauchamp, M. S., Cox, R. W., \& DeYoe, E. A. (1997). Graded effects 
of spatial and featural attention on human area MT and associated motion processing areas. Journal of Neurophysiology, 78, 516-520.

Botvinick, M. M., Braver, T. S., Barch, D. M., Carter, C. S., \& Cohen, J. D. (2001). Conflict monitoring and cognitive control. Psychological Review, 108, 624-652.

Brett, M., Anton, J.-L., Valabregue, R., \& Poline, J.-B. (2002). Region of interest analysis using an SPM toolbox. Abstract presented at the 8th International Conference on Functional Mapping of the Human Brain, Sendai, Japan.

Brown, J. W., \& Braver, T. S. (2007). A computational model of risk, conflict, and individual difference effects in the anterior cingulate cortex. Brain Research, 1202, 99-108.

Carter, C. S., Braver, T. S., Barch, D. M., Botvinick, M. M., Noll, D., \& Cohen, J. D. (1998). Anterior cingulate cortex, error detection, and the online monitoring of performance. Science, $\mathbf{2 8 0}$, 747-749.

Corbetta, M., Shulman, G. L., Miezin, F. M., \& Petersen, S. E. (1995). Superior parietal cortex activation during spatial attention shifts and visual feature conjunction. Science, 270, 802-805.

Cocosco, C. A., Kollokian, V., Kwan, R. K.-S., \& Evans, A. C. (1997). BrainWeb: Online interface to a 3D MRI simulated brain database. In Proceedings of the 3rd International Conference on Functional Mappping of the Human Brain. Copenhagen.

Ditterich, J., Mazurek, M. E., \& Shadlen, M. N. (2003). Microstimulation of visual cortex affects the speed of perceptual decisions. Nature Neuroscience, 6, 891-898

Dubner, R., \& ZeKI, S. M. (1971). Response properties and receptive fields of cells in an anatomically defined region of the superior temporal sulcus in the monkey. Brain Research, 35, 528-532.

Egner, T., Delano, M., \& Hirsch, J. (2007). Separate conflict-specific cognitive control mechanisms in the human brain. NeuroImage, $\mathbf{3 5}$, 940-948.

EgNer, T., \& Hirsch, J. (2005). Cognitive control mechanisms resolve conflict through cortical amplification of task-relevant information. Nature Neuroscience, 8, 1784-1790.

Grefkes, C., \& Fink, G. R. (2005). The functional organization of the intraparietal sulcus in humans and monkeys. Journal of Anatomy, 207, 3-17.

Huk, A. C., Dougherty, R. F., \& Heeger, D. J. (2002). Retinotopy and functional subdivision of human areas MT and MST. Journal of Neuroscience, 22, 7195-7205.

Kerns, J. G., Cohen, J. D., MacDonald, A. W., III, Cho, R. Y., Stenger, V. A., \& CARTER, C. S. (2004). Anterior cingulate conflict monitoring and adjustments in control. Science, 303, 1023-1026.

KoRnBLUM, S. (1994). The way irrelevant dimensions are processed depends on what they overlap with: The case of Stroop- and Simon-like stimuli. Psychological Research, 56, 130-135.

Lee, K. M., Chang, K. H., \& RoH, J. K. (1999). Subregions within the supplementary motor area activated at different stages of movement preparation and execution. NeuroImage, 9, 117-123.

Liston, C., Matalon, S., Hare, T. A., Davidson, M. C., \& Casey, B. J. (2006). Anterior cingulate and posterior parietal cortices are sensitive to dissociable forms of conflict in a task-switching paradigm. Neuron, 50, 643-653.

LiU, X., Banich, M. T., Jacobson, B. L., \& Tanabe, J. L. (2004). Common and distinct neural substrates of attentional control in an integrated Simon and spatial Stroop task as assessed by event-related fMRI. NeuroImage, 22, 1097-1106.

LuCK, S. J., \& ForD, M. A. (1998). On the role of selective attention in visual perception. Proceedings of the National Academy of Sciences, 95, 825-830.

Macaluso, E., Frith, C. D., \& Driver, J. (2007). Delay activity and sensory-motor translation during planned eye or hand movements to visual or tactile targets. Journal of Neurophysiology, 98, 3081-3094.

MacDonald, A. W., III, Cohen, J. D., Stenger, V. A., \& Carter, C. S. (2000). Dissociating the role of the dorsolateral prefrontal and anterior cingulate cortex in cognitive control. Science, 288, 1835-1838.
Milham, M. P., Banich, M. T., Webb, A., Barad, V., Cohen, N. J., Kramer, A. F., ET AL. (2001). The relative involvement of anterior cingulate and prefrontal cortex in attentional control depends on nature of conflict. Cognitive Brain Research, 12, 467-473.

Nelson, J. K., Reuter-Lorenz, P. A., Sylvester, C.-Y., Jonides, J., \& Sмiтн, E. E. (2003). Dissociable neural mechanisms underlying response-based and familiarity-based conflict in working memory. Proceedings of the National Academy of Sciences, 100, 11171-11175.

Palmer, J., Huk, A. C., \& Shadlen, M. N. (2005). The effect of stimulus strength on the speed and accuracy of a perceptual decision. Journal of Vision, 5, 376-404.

Posner, M. I., \& Dehaene, S. (1994). Attentional networks. Trends in Neurosciences, 17, 75-79.

RidDERINKHOF, K. R., Ullsperger, M., Crone, E. A., \& Nieuwenhuis, S. (2004). The role of the medial frontal cortex in cognitive control. Science, 306, 443-447.

Roitman, J. D., \& Shadlen, M. N. (2002). Response of neurons in the lateral intraparietal area during a combined visual discrimination reaction time task. Journal of Neuroscience, 22, 9475-9489.

Salzman, C. D., Murasugi, C. M., Britten, K. H., \& Newsome, W. T. (1992). Microstimulation in visual area MT: Effects on direction discrimination performance. Journal of Neuroscience, 12, 2331-2355.

Shadlen, M. N., \& Newsome, W. T. (2001). Neural basis of a perceptual decision in the parietal cortex (area LIP) of the rhesus monkey. Journal of Neurophysiology, 86, 1916-1936.

TAlairach, J., \& Tournoux, P. (1988). Co-planar stereotaxic atlas of the human brain: 3-dimensional proportional system-An approach to cerebral imaging. Stuttgart: Thieme.

Treue, S., \& Martinez Trujillo, J. C. (1999). Feature-based attention influences motion processing gain in macaque visual cortex. Nature, 399, 575-579.

van Veen, V., \& CARTer, C. S. (2005). Separating semantic conflict and response conflict in the Stroop task: A functional MRI study. NeuroImage, 27, 497-504.

van Veen, V., Cohen, J. D., Botvinick, M. M., Stenger, V. A., \& CARTER, C. S. (2001). Anterior cingulate cortex, conflict monitoring, and levels of processing. NeuroImage, 14, 1302-1308.

Verbruggen, F., Notebaert, W., Liefooghe, B., \& VANDierendoncK, A. (2006). Stimulus- and response-conflict-induced cognitive control in the flanker task. Psychonomic Bulletin \& Review, 13, 328-333.

Watson, J. D., Myers, R., Frackowiak, R. S., Hajnal, J. V., Woods, R. P., Mazziotta, J. C., ET AL. (1993). Area V5 of the human brain: Evidence from a combined study using positron emission tomography and magnetic resonance imaging. Cerebral Cortex, 3, 79-94.

Weissman, D. H., Giesbrecht, B., Song, A. W., Mangun, G. R., \& WoldorfF, M. G. (2003). Conflict monitoring in the human anterior cingulate cortex during selective attention to global and local object features. NeuroImage, 19, 1361-1368.

Wendelken, C., \& Shastri, L. (2005). Connectionist mechanisms for cognitive control. Neurocomputing, 65-66, 663-672.

ZEKI, S. M. (1974). Functional organization of a visual area in the posterior bank of the superior temporal sulcus of the rhesus monkey. Journal of Physiology, 236, 549-573.

\section{NOTE}

1. We consider "unselected," rather than "incorrect," responses here because it is the selected response, not necessarily the correct response, that should be associated with greater activation (since stronger activation of a response representation leads to selection of that response). And it is an increase in the smaller of two inputs (i.e., associated with the unselected response) that should have the greater positive effect on conflict. These labels are equivalent, of course, for correct trials, but they are flipped on incorrect trials.

(Manuscript received October 13, 2008; revision accepted for publication July 31, 2009.) 